\title{
(3) 2001110.375
}

\section{SOLID INFLAMMABILITY BOUNDARY AT LOW-SPEED (SIBAL)}

\author{
James T'ien*, Paul Ferkul ${ }^{+}$, Kurt Sacksteder ${ }^{\#}$, Hsin-Yi Shih*, Amit Kumar*, \\ Julie Kleinhenz*, Hasan Bedir*, Richard Pettegrew ${ }^{+}$, Nancy Piltch ${ }^{\sharp}$ and David Frate ${ }^{\#}$ \\ *Case Western Reserve University, Cleveland, Ohio 44106 \\ National Center for Microgravity Research, Cleveland, Ohio 44135 \\ "NASA Glenn Research Center, Cleveland, Ohio 44135
}

\section{INTRODUCTION}

This research program is concerned with the effect of low-speed, concurrent flow on the spreading and extinction processes of flames over solid fuels. The primary objective is to verify the theoretically predicted extinction boundary, using oxygen percentage and flow velocity as coordinates. Of particular interest are the low-speed quenching limits and the existence of the critical oxygen flammability limit. Detailed flame spread characteristics, including flame spread rate, flame size, and flame structure are sought. Since the predicted flame behavior depends on the inclusion of flame and surface radiation, the measured results will also be used to assess the importance of radiative heat transfer by direct comparison to a comprehensive numerical model.

The solid fuel used in this experiment is a custom-made fabric consisting of a $1: 1$ blend of cotton and fiberglass. This choice was made following an extensive search to yield a material with favorable properties, namely, rollability, non-cracking behavior during combustion, strength after combustion, and flammability in a range of oxygen limits permissible within the Combustion Integrated Rack (CIR) on the International Space Station. At the present time, an effort is being made to characterize both the radiative properties of the fuel and the flame spreading behavior in normal gravity at reduced pressure. These will provide a basis for comparison with the microgravity results as well as aid in bracketing the anticipated flammability boundary for the flight experiment.

An overview of recent work, with emphasis on theoretical results, is presented below.

\section{THREE-DIMENSIONAL FLAME SPREAD MODEL}

A previously established two-dimensional laminar flame spread model over a thin solid in low-speed concurrent flow has been extended to three-dimensions. The model consists of the full Navier-Stokes equations for the conservation of mass, momentum energy and species in the gasphase. The species equations are for the fuel vapor, oxygen, carbon dioxide and water vapor. A onestep, second-order, finite-rate Arrhenius reaction between fuel vapor and oxygen is assumed. The solid obeys a zeroth-order pyrolysis relation and is thermally and aerodynamically thin. Both surface and gas radiation are included in the model. However, the coupled computation with the threedimensional gas-phase radiative transfer equation is very time-consuming. Thus far, the computed solutions are restricted to the case with surface radiative loss only.

Computations have been performed with the solid fuel inside a three-dimensional flow tunnel. In the first configuration examined, the tunnel height is fixed at $10 \mathrm{~cm}$, and the solid fuel is placed at the middle height. The solid is fed into the tunnel at the steady burnout rate (only steady solutions are being sought). Laterally, the fuel spans the entire tunnel width (i.e., from wall to wall). The tunnel width (which is identical to the solid fuel width) is a parameter in the numerical study (varied from $2 \mathrm{~cm}$ to infinity).

In addition to the fuel width, the incoming flow velocity and the oxygen percentage are varied. The fuel burnout is fixed at $6 \mathrm{~cm}$ from the tunnel entrance. In this configuration, several three-dimensional effects due to the presence of the tunnel walls are observed. First, the wall 
changes the velocity profiles due to the no-slip boundary condition. Second, the cold sidewalls conduct heat away from the flame, which produces heat loss and a quenched layer. Third, the quenched layer near the walls creates a space for oxygen side diffusion to the flame.

At higher flow velocities (away from limit cases), the three-dimensional effect is mostly heat transfer in origin, as the flames spread faster for wider samples. However, near the low-speed, lowoxygen limit, many unexpected results appear. The competition between wall heat loss, wall quenching, and oxygen side diffusion creates a number of unusual phenomena for the near-limit flames. These include faster spread for narrower samples and an extension of flammability beyond the two-dimensional limit for moderately narrow samples (about $4 \mathrm{~cm}$ wide for the specified conditions) [1,2]. The key mechanism responsible for these unusual phenomena appears to be the side diffusion of oxygen, which finds its way to the flame through quenched wall layers. Oxygen side diffusion strengthens the oxygen-starved near-limit flames. It also pushes the flames closer to the solid surface, which increases the flame heat feedback and the spread rate.

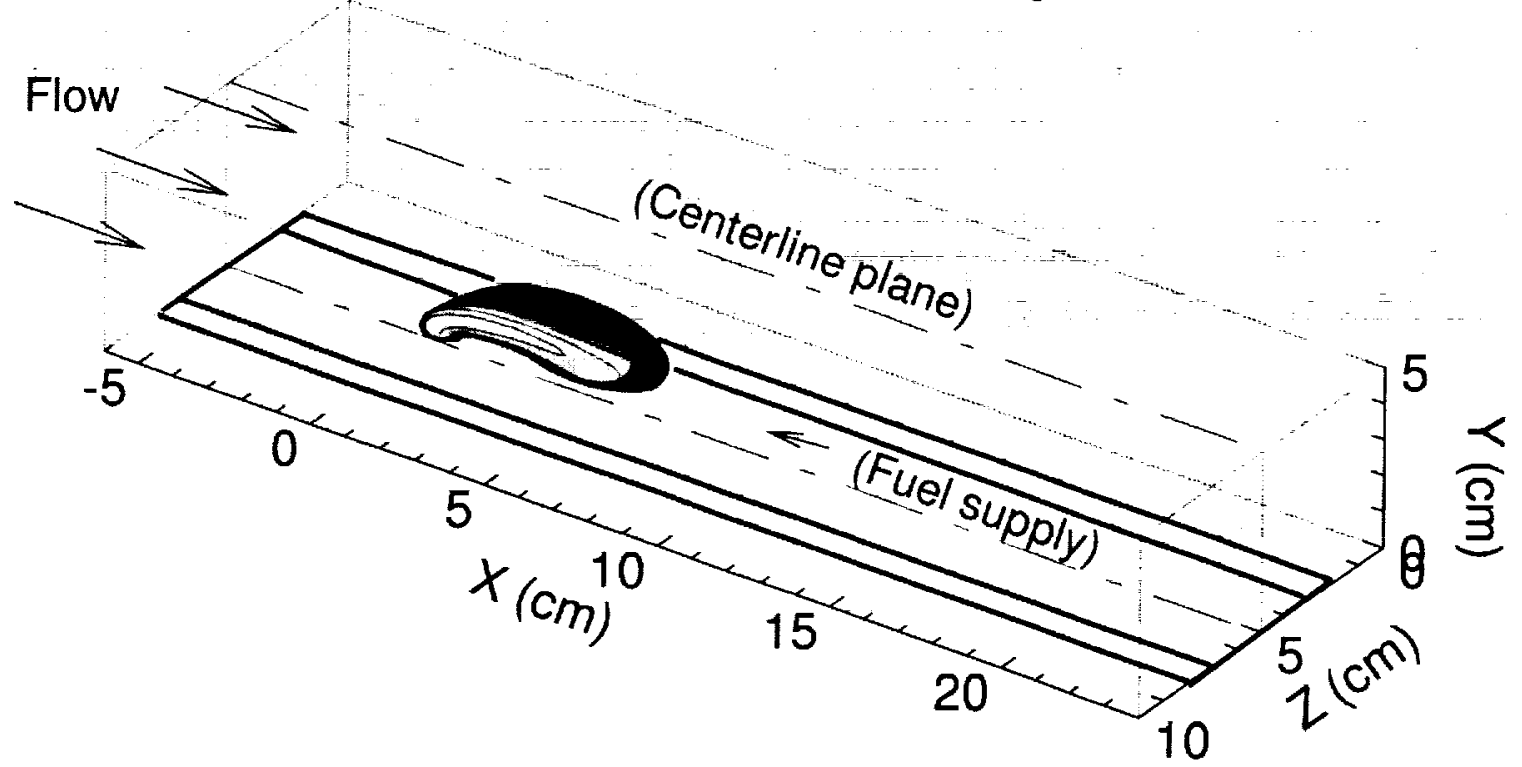

Figure 1. Schematic of the three-dimensional flame in the flow tunnel. Upper half of the tunnel configuration is shown. The 4-cm wide solid fuel $(\mathrm{Y}=0)$ is fitted with a $1-\mathrm{cm}$ inert strip on each side. The computed flame (only half of the upper flame) is shown stabilized over the solid where the flame base position $(X=0)$ is $6 \mathrm{~cm}$ away from the tunnel entrance. A forced oxidizer flow is imposed at the entrance and the solid fuel is fed into the flame.

In the proposed flight experiment, the tunnel flow cross section is fixed $(10 \mathrm{~cm} \times 10 \mathrm{~cm})$ but the fuel sample width can be varied. In addition, to facilitate the translation of the sample and to eliminate edge effects, two inert strips are mounted on the sample sides (fig. 1). This realistic geometry is the second configuration examined numerically. With the inert strips, the flame spread rate increases due to the decrease of the wall heat loss and the promotion of the oxygen side diffusion. To demonstrate the modeling capability, Figures 2 and 3 give an illustration of the types of results computed.

Figure 2 shows the non-dimensional solid thickness and surface temperature contours of a 4 cm wide solid fuel (thin cellulosic sheets with $20 \%$ noncombustible material) in $5 \mathrm{~cm} / \mathrm{s}, 15 \% \mathrm{O}_{2}$ flow. The pyrolysis front (thickness $\sim 0.95$ ) and bumout front are seen clearly to exhibit a threedimensional effect. This three-dimensional feature is even more pronounced in the preheat zone as shown by the surface temperature contours. It is expected from the consideration of the length-towidth ratio. The curvature of the contours in the downstream region is opposite to those in the 
upstream flame base region. It is also noteworthy that in this calculation, the area thermal inertia of the inert strips is assumed to be 100 times that of the fuel. Consequently, the strip temperature is low. The strip thermal inertia is a parameter in the model and in the experiment.

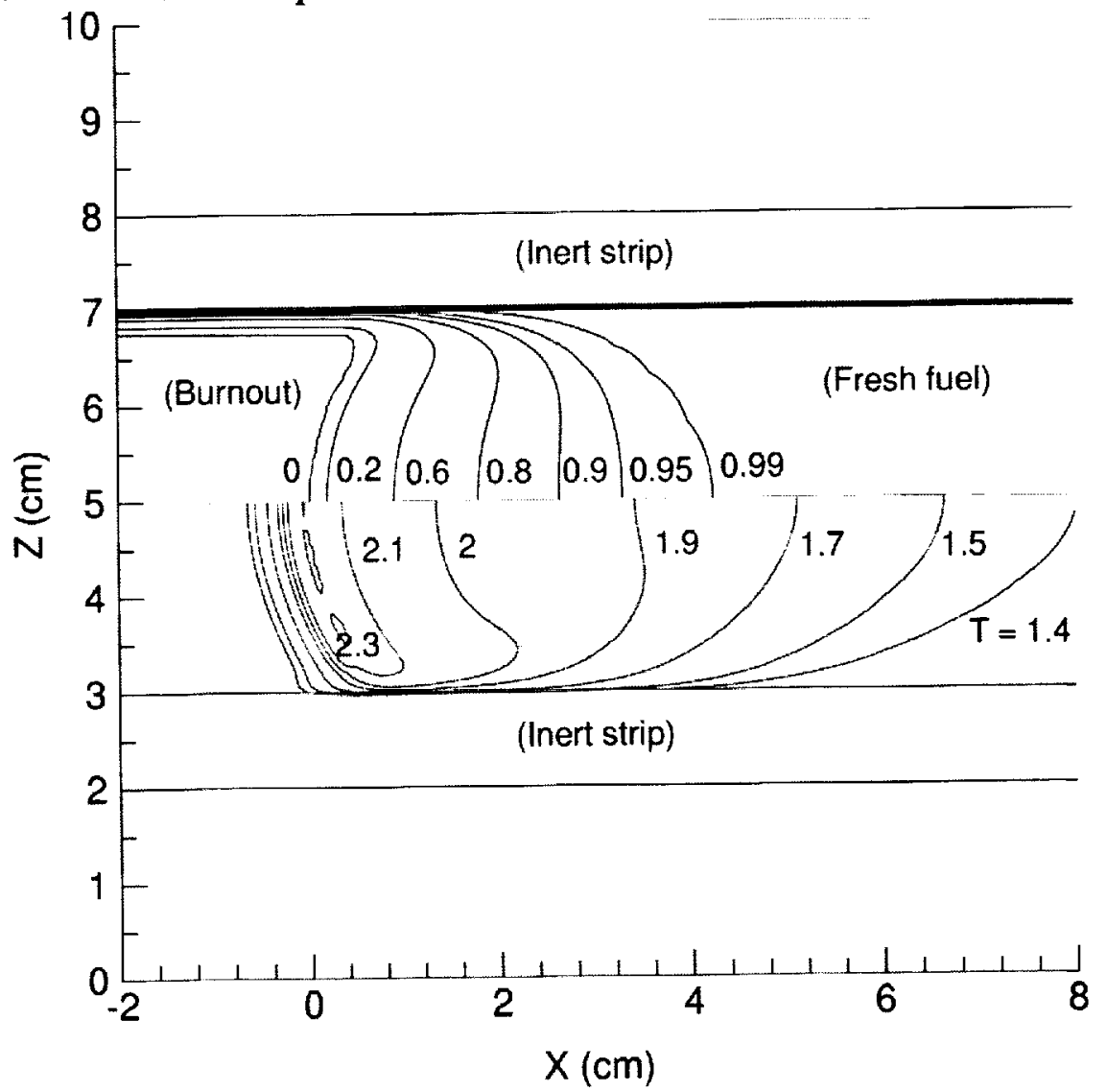

Figure 2. The solid thickness (upper half) and temperature contours (lower half, 1 unit $=300 \mathrm{~K}$ ).

Figure 3 presents the projections of the streamlines and velocity vectors on the plane $8.5 \mathrm{~mm}$ above the solid surface. Also shown are the oxygen mass flux vectors, temperature contours, and fuel reaction rate contours. Note that due to thermal expansion, the hot temperature contours (e.g., T $=2$ ) are extended beyond the width of the sample, consistent with experimental observations. Also note that side diffusion of oxygen is very pronounced near the flame base region. These computed results will provide guidance on the types of flame structure measurements that should be performed in the flight experiment.

\section{OPPOSED AND CONCURRENT SPREADING FLAMES COMPARISON}

In order to fully understand the relationship between flame spread in concurrent and in opposed flows, a two-dimensional opposed-flow model with the same assumptions as the twodimensional concurrent spread model is developed [3]. Both contain gas radiation.

Flammability limits and spread rates are computed using oxygen percentage and free stream velocity as parameters. The opposed flow result is compared to that of concurrent flow. In both cases, the flammability boundary is a typical U-shaped curve which consists of two branches, namely a low-speed quenching branch which is due to radiative losses from both the gas phase and the fuel surface, and a high-speed blowoff branch which is due to inadequate flow residence time. Although 
qualitatively similar, the comparison of the two flammability boundaries shows that there is a crossover. When the flow velocity is sufficiently low, the flame in opposed flow can be more flammable, while at higher flow velocities the flame in concurrent flow is more flammable. This is an unusual and intriguing discovery but can be explained based on the relative flow velocity with respect to the flame.

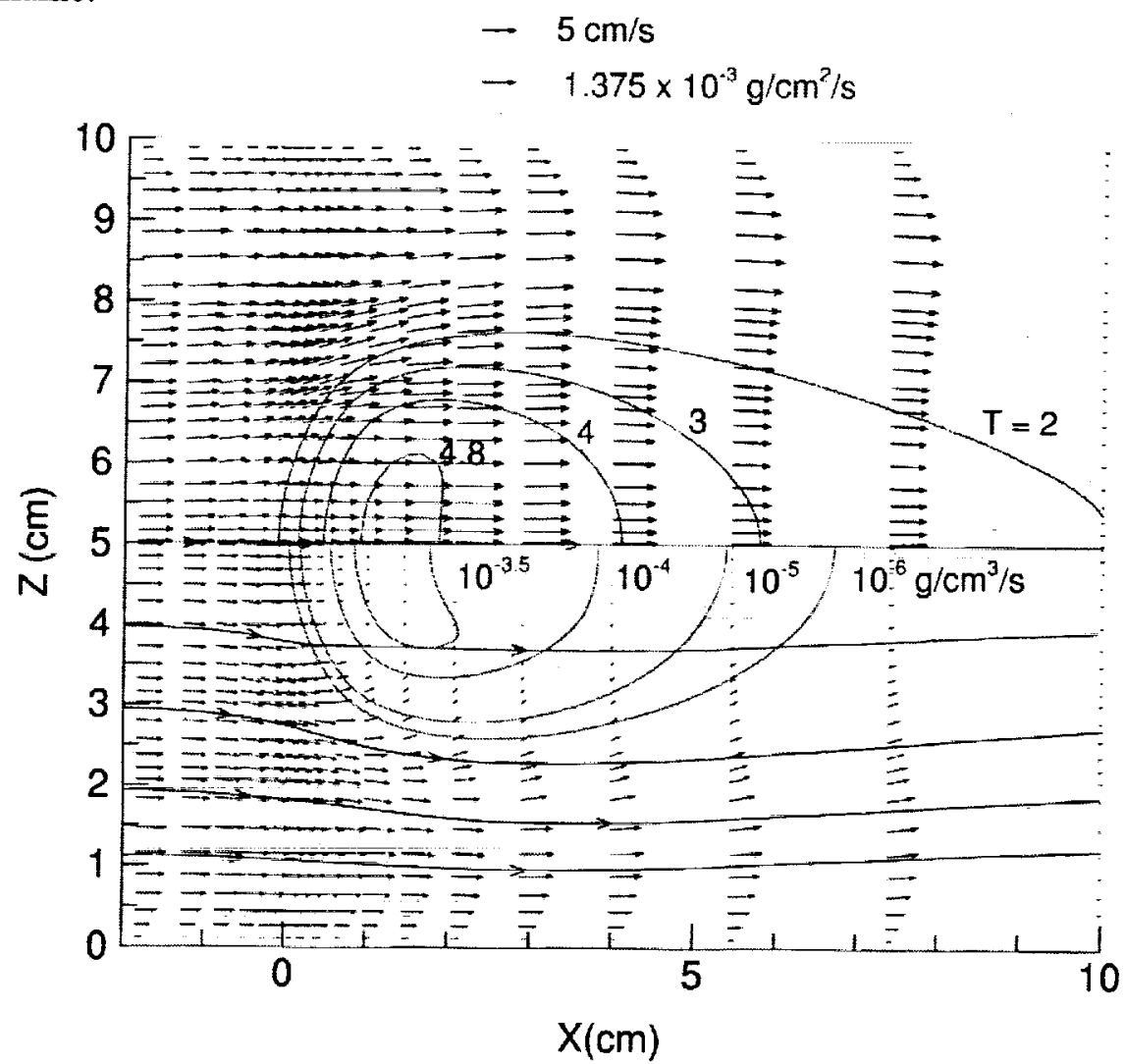

Figure 3. The detailed flame structure on the plane at height $\mathrm{Y}=8.5 \mathrm{~mm}$ above the solid. Upper half: velocity vectors, temperature contours $(1$ unit $=300 \mathrm{~K})$. Lower half: velocity streamlines, fuel reaction rate contours, and oxygen mass flux vectors. The vectors are projections on this plane.

The flame-spread rates in opposed and concurrent flow exhibit different trends. The flamespread rate in concurrent flow is approximately proportional to the flow velocity while in opposed flow the variation of spread rate with flow velocity has a maximum at an intermediate flow velocity and drops near both the high and the low velocity limits. The two spread rate curves again can crossover. The spread rates for opposed flow can be higher than those for concurrent flow when the flow velocity is sufficiently low.

\section{REFERENCES}

1. Shih, H. Y.: "A Three-Dimensional Model of Flame Spread Over a Thin Solid in Low-Speed Concurrent Flow," Ph.D. Dissertation, Case Western Reserve University, Cleveland, Ohio (January 2000).

2. Shih, H. Y. and T'ien, J. S.: "Modeling Concurrent Flame Spread Over a Thin Solid in a LowSpeed Flow Tunnel," Proceedings of the Combustion Institute 28, to appear (2000).

3. Kumar, A., Shih, H. Y. and T'ien, J. S.: "A Comparison of Extinction Limits and Spreading Rates in Opposed and Concurrent Spreading Flames Over Thin Solids," Presented at the $2^{\text {nd }}$ Joint Meeting of the US Sections of the Combustion Institute (March 2001). 